\title{
2016 Manuscript Reviewers
}

The following individuals assisted in the manuscript peer review process on one or more occasions between 19 November 2015 and 22 November 2016. We are deeply grateful for their time and efforts on our behalf.

\begin{tabular}{|c|c|c|c|}
\hline Aars, J. & Dumond, D.E. & Krebs, C.J. & Ritchie, R.J. \\
\hline Acorn, J. & Dunmall, K. & Kwok, R. & Rizzolo, D.J. \\
\hline Adler, P.H. & Durner, G.M. & Kylin, H. & Robertson, G.J. \\
\hline Aerts, L. & Eisaguirre, J.M. & Lammers, R.B. & Rockwell, R.F. \\
\hline Anstey, R. & Epstein, H.E. & Leafloor, J.O. & Rode, K.D. \\
\hline Appelt, M. & Feder, H.M. & Lecomte, N. & Roy-Léveillée, $\mathrm{P}$. \\
\hline Arp, C.D. & Fischbach, A.S. & Lofthouse, S. & Ruthrauff, D.R. \\
\hline Ashjian, C.J. & Fischer, J. & Loring, S. & Ryan, K. \\
\hline Bain, A. & Fitzhugh, W.W. & Lovvorn, J.R. & Sabin, J. \\
\hline Baird, P.A. & Flint, P.L. & Lowry, L.F. & Safine, D.E. \\
\hline Baker, B. & Forbes, D.L. & Lunn, N.J. & Sahanatien, V. \\
\hline Balasse, M. & Forbes, V. & Madsen, C.K. & Sandlos, J. \\
\hline Battersby, W. & Foulke, T.K. & Magdanz, J.S. & Scarre, C.J. \\
\hline Batzli, G.O. & Friesen, T.M. & Makynen, M. & Schindler, D.E. \\
\hline Beattie, $\mathrm{O}$. & Frink, L. & Mallory, M.L. & Schledermann, P. \\
\hline Behe, C. & Froese, D. & Marcoux, M. & Schmidt, J. \\
\hline Betts, M.W. & Fuglei, E. & Mason, O.K. & Schmidt, J.I. \\
\hline Binnema, T. & Fulton, T. & McClelland, J.W. & Schott, S. \\
\hline Bjerck, H.B. & Galloway, T. & Melling, $\mathrm{H}$. & Seger, J. \\
\hline Blankholm, H.P. & Gerard, $\mathrm{P}$. & Merizon, R.A. & Sharp, M.J. \\
\hline Blomqvist, $\mathrm{S}$. & Gill, R.E., Jr. & Merkel, F.R. & Sheehy, P.J.A. \\
\hline Boertmann, D. & Greenway, G. & Meyer, K. & Sherman, K. \\
\hline Boisvert, L.N. & Grimwood, B.S.R. & Michelutti, N. & Sirovic, A.S. \\
\hline Braem, N.M. & Guly, H. & Millar, K. & Skarin, A. \\
\hline Britton, K. & Hambrecht, G. & Moen, J. & Sørensen, M. \\
\hline Brown, C.L. & Hannus, A. & Montgomerie, R. & Spiers, K. \\
\hline Brown, C.T. & Harper, S. & Natcher, D.C. & Staples, K. \\
\hline Brown, T. & Hart, S.J. & Neuvonen, S. & Steinnes, E. \\
\hline Brunet, N.D. & Harwood, L.A. & Obbard, M.E. & Stern, P. \\
\hline Bueddefeld, J. & Heidt, D. & Odess, D. & Stewart, R.E.A. \\
\hline Burn, C.R. & Henri, D. & Odgaard, U. & Stuhl, A. \\
\hline Cahill, J. & Hewlett, J.P. & Odland, J.O. & Suydam, R.S. \\
\hline Cahoon, S. & Higdon, J.W. & Ogden, A.R. & Sydnes, A.K. \\
\hline Carey, M. & Holen, D.L. & Park, R.W. & Tape, K.D. \\
\hline Cavell, J. & Holly, D.H. & Pearce, T. & Tester, F. \\
\hline Chan, L.H.M. & Hood, B. & Peters, D.L. & Therkildsen, O.R. \\
\hline Chapin, F.S. & Hoppe, K.A. & Pettis, H. & Thomas, P.O. \\
\hline Chisholm, D. & Howell, S.E.L. & Pinard, C. & Van de Noort, R. \\
\hline Citta, J.J. & Huettmann, F. & Poelzer, G. & Van Parijs, S. \\
\hline Clark, C.W. & Huntington, H.P. & Pomerleau, C. & Voorhees, H. \\
\hline Clark, D.A. & Ikis, D. & Potter, R.A. & Walls, M. \\
\hline Clarke, J.T. & Jay, C.V. & Proctor, A. & Walsh, C.J. \\
\hline Collings, P.F. & Jensen, E.L. & Quakenbush, L.T. & Wamsley, D.W. \\
\hline Colpaert, A. & Johnson, R. & Rajdev, V. & Wells, P. \\
\hline Corbett, D. & Johnston, V. & Rasic, J. & Wesche, S.D. \\
\hline Cox, S. & Johnstone, J.F. & Ravens, T.M. & Westdal, K.H. \\
\hline Cronin, M.A. & Jones, J. & Raynolds, M.K. & Wexler, L. \\
\hline Curasi, S.R. & Jorgenson, $\mathrm{T}$. & Reimers, E. & Whiting, S.J. \\
\hline Currie, D.C. & Kellett, D.K. & Reneerkens, J.W.H. & Wigda, C. \\
\hline Dann, T.H. & Kikkert, P. & Reuther, J.D. & Wong, P. \\
\hline Dawson, P.C. & Kinsman, N. & Rice, A.N. & Young, M.G. \\
\hline Desrosiers, P.M. & Knecht, R.A. & Richard, P. & Yurkowski, D. \\
\hline Dixon, E.J. & Kovacs, K.M. & Richardson, W.J. & Zazula, G.D. \\
\hline Dufraisse, A. & & & Zutter, C. \\
\hline
\end{tabular}

